\title{
Development of Root Phenotyping Platforms for Identification of Root Architecture Mutations in EMS-Induced and Low-Path-Sequenced Sorghum Mutant Population
}

\author{
Viktor Tishchenko ${ }^{1 *}$, Mingli Wang ${ }^{2 *}$, Zhanguo Xin ${ }^{3}$, Melanie Harrison ${ }^{2}$ \\ ${ }^{1}$ College of Agricultural and Environmental Sciences, University of Georgia, Griffin, GA, USA \\ ${ }^{2}$ USDA-ARS, Plant Genetic Resources Conservation Unit, Griffin, GA, USA \\ ${ }^{3}$ USDA-ARS, Plant Stress and Germplasm Development Research Unit, Lubbock, TX, USA \\ Email: *viktort@uga.edu, ^mingli.wang@usda.gov
}

How to cite this paper: Tishchenko, V., Wang, M.L., Xin, Z.G. and Harrison, M. (2020) Development of Root Phenotyping Platforms for Identification of Root Architecture Mutations in EMS-Induced and Low-Path-Sequenced Sorghum Mutant Population. American Journal of Plant Sciences, $11,838-850$.

https://doi.org/10.4236/ajps.2020.116060

Received: February 7, 2019

Accepted: June 25, 2020

Published: June 28, 2020

Copyright $\odot 2020$ by author(s) and Scientific Research Publishing Inc. This work is licensed under the Creative Commons Attribution International License (CC BY 4.0).

http://creativecommons.org/licenses/by/4.0/

\section{(c) (i) Open Access}

\begin{abstract}
Sorghum's natural adaptation to a wide range of abiotic stresses provides diverse genetic reserves for potential improvement in crop stress tolerance. Growing interest in sorghum research has led to the expansion of genetic resources though establishment of the sorghum association panel (SAP), generation of mutagenized populations, and recombinant inbred line (RIL) populations, etc. Despite rapid improvement in biotechnological tools, lack of efficient phenotyping platforms remains one of the major obstacles in utilizing these genetic resources. Scarcity of efforts in root system phenotyping hinders identification and integration of the superior root traits advantageous to stress tolerance. Here, we explored multiple approaches in root phenotyping of an ethyl methanesulfonate (EMS)-mutagenized sorghum population. Paper-based growth pouches (PGP) and hydroponics were employed to analyze root system architecture (RSA) variations induced by mutations and to test root development flexibility in response to phosphorus deficiency in early growing stages. PGP method had improved capabilities compared to hydroponics providing inexpensive, space-saving, and high-throughput phenotyping of sorghum roots. Preliminary observation revealed distinct phenotypic variations which were qualitatively and quantitatively systemized for association analysis. Phenotypes/ideotypes with root architecture variations potentially correlated with $\mathrm{Pi}$ acquisition were selected to evaluate their contribution to P-efficiency (PE). Sand mixed with P-loaded activated alumina substrate (SAS) provided closely to natural but still controlled single-variable
\end{abstract}


conditions with regulated Pi availability. Due to higher labor and cost input we propose SAS to be used for evaluating selected sorghum candidates for PE. The ability of rapidly screening root phenotypes holds great potential for discovering genes responsible for relevant root traits and utilizing mutations to improve nutrient efficiency and crop productivity.

\section{Keywords}

Sorghum, Mutagenized, Phenotyping, Root System Architecture, P Efficiency and Deficiency, Nutrients

\section{Introduction}

Sorghum is known for its tolerance to drought and nutrient deficiencies due to root system adaptations [1] [2]. However, root architecture traits contributing to superior performance and their genetic regulators have not been sufficiently studied. In spite of the rapid progress in biotechnology and expanding molecular/genomic tools, utilizing advantageous root traits remains challenging due to limited root phenotyping progress. Underground allocation, soil multivariable nature, and complex root interaction with environmental factors restrict root study. Field phenotyping methods (such as shovelomics or rhizotron) are laborious, time-consuming and not as efficient as aboveground plant observation [3] [4]. Numerous controlled-environment approaches employ various artificial media of nutrient solution, agarose gel chambers, or sand-alumina media provide improved-phenotyping capabilities. Recently, due to the need for identification of quantitative trait loci (QTL) and genes associated with root traits, the emphasis has shifted to systems that can provide inexpensive, efficient, high throughput phenotyping such as paper pouches [5] [6] [7]. While some of these methods have been used, only few were employed in sorghum root phenotyping, narrowly focused on specific traits [8] and left large unexplored areas of root development. In addition, the availability of characterized sorghum genetic resources (such as recombinant inbred lines and mutagenized populations) is still limited. Chemically mutagenized sorghum population offers great opportunity in discovering sorghum root morphological variation and identification of candidate genes [9]. Reported diverse assortment and frequency of the mutant root morphological variations make it a valuable resource for fulfilling belowground phenotyping and targeting useful morphological and agronomic traits [10]. Here, we explored multiple approaches in developing universal, systemized, and efficient high-throughput root phenotyping platforms for assessing large populations such as an EMS-mutagenized population (MP). To characterize root architecture of the total MP, we tested multiple approaches for their ability to characterize root architecture, to distinguish candidates with traits of interest, and to evaluate the role of specific root traits on sorghum performance in modulated natural/close to natural $\mathrm{P}$ deficient environment conditions. In addition, me- 
thods were tested to evaluate plant flexibility in root development response to $\mathrm{P}$ and other nutrient deficiency.

\section{Materials and Methods}

In total, 254 lines of the EMS-mutagenized and low-path-sequenced sorghum population $\left(\mathrm{M}_{3}\right.$ generation) were acquired from the USDA-ARS, PSGDRU, Lubbock, TX. Seeds were pretreated with Arazon 705 and germinated on germination paper rolls for two days at $30^{\circ} \mathrm{C}$ in the dark. Germinated seeds were then transferred to a growing media [either hydroponics, or paper-based growth poches (PGP), or sand mixed with P-loaded activated alumina substrate (SAS)]. Seedlings were grown at the Georgia Envirotron research facility in the growth chamber (PGW36, Conviron) at $27 / 24^{\circ} \mathrm{C}$ day/night with 12 hours photoperiod, photosynthetically active radiation $(\mathrm{PAR})=600 \mu \mathrm{mol} \cdot \mathrm{m}^{-2} \cdot \mathrm{s}^{-1}$.

\subsection{Growing Techniques for Root Phenotyping}

In both hydroponic and PGP experiments, Magnavaca's nutrient solution was used [11] [12] with some modifications. Composition of the nutrient solution: $\left(\mathrm{mmol} \cdot \mathrm{L}^{-1}\right): 7.556 \mathrm{Ca}\left(\mathrm{NO}_{3}\right)_{2} \cdot 4 \mathrm{H}_{2} \mathrm{O}, 2.787 \mathrm{NH}_{4} \mathrm{NO}_{3}, 1.796 \mathrm{KCl}, 1.818 \mathrm{~K}_{2} \mathrm{SO}_{4}, 1.752$ $\mathrm{KNO}_{3}, 1.555 \mathrm{Mg}\left(\mathrm{NO}_{3}\right)_{2} \cdot 6 \mathrm{H}_{2} \mathrm{O}, \mathrm{Fe}-\mathrm{EDDHA}$ (Sprint 138), $\mathrm{KH}_{2} \mathrm{PO}_{4}, 1.8 \times 10^{-2}$ $\mathrm{MnCl}_{2} \cdot 4 \mathrm{H}_{2} \mathrm{O}, 4.9 \times 10^{-2} \mathrm{H}_{3} \mathrm{BO}_{3}, 4.6 \times 10^{-3} \mathrm{ZnSO}_{4} \cdot 7 \mathrm{H}_{2} \mathrm{O}, 1.2 \times 10^{-3} \mathrm{CuSO}_{4} \cdot 5 \mathrm{H}_{2} \mathrm{O}$, $1.6 \times 10^{-3} \mathrm{Na}_{2} \mathrm{MoO}_{4} \cdot 2 \mathrm{H}_{2} \mathrm{O}$ Seedlings were evaluated visually and scanned with Plstek OpticPro A320 scanner that generated high quality images in order to distinguish, quantify, and classify root types. Images were analyzed with RootReader2D software as outlined by R. Clark [13]. Total root length (TRL), primary root length (PRL), total length of all lateral roots (LRL), lateral root number (LRN), and six longest lateral roots length (6LLR) were recorded.

\subsubsection{Hydroponics}

Germinated seedlings were placed in seedling caps with roots submerged into 0.5 Magnavaca's nutrient solution (Figure 1(A)). Plants were grown for seven days and scanned for generating images.

\subsubsection{Paper-Based Growth Pouches (PGP)}

Germinated seedlings were transferred to a growth pouch system developed by Hund [5] with some minor modifications (Figure 1(B) and Figure 1(C)). Growth pouches with seeds were positioned into plastic containers so that the lowest $2 \mathrm{~cm}$ of the paper was submerged in a 0.5 Magnavaca's nutrient solution and grown for seven days.

The $\mathrm{Pi}$ availability response experiment was conducted with the same parameters except various phosphorus concentrations were utilized. Seeds from BTx623 with similar size as the check were selected and germinated. Plants were transferred to growth pouches and grown for seven days at the following levels of $\mathrm{KH}_{2} \mathrm{PO}_{4}: 0,3,25,45$, and $65 \mathrm{PO}_{4}^{3-}\left(\mu \mathrm{mol} \cdot \mathrm{L}^{-1}\right)$ at 0.5 and 1.0 strength Magnavaca's nutrient solution. A set of plants were also grown in deionized water as the control. 


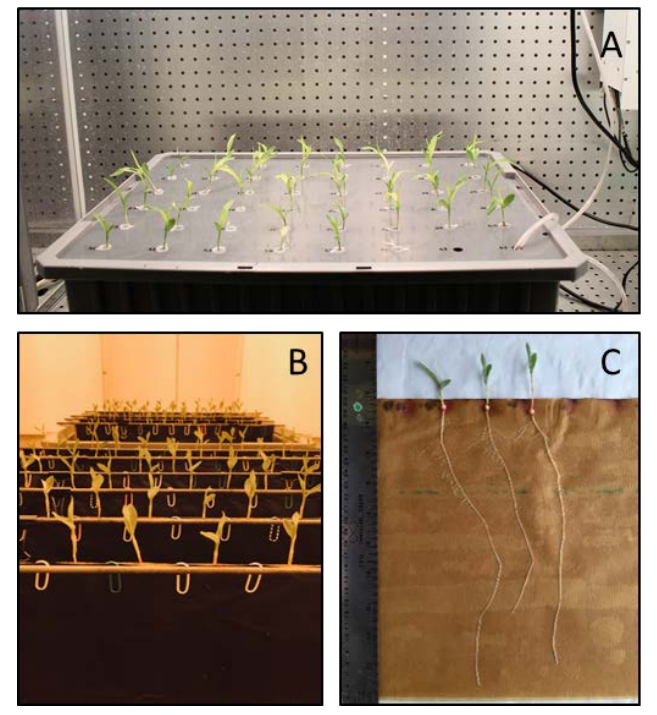

Figure 1. Images of the liquid culture method. Hydroponics (A) and paper growth pouches (B, shoots; C, roots).

\subsection{Sand Culture Amended with P-Loaded Activated Alumina (SAS)}

SAS medium was composed of predominantly quartz sand ( $98 \%)$ mixed with P-loaded activated alumina $\left(\mathrm{Al}_{2} \mathrm{O}_{3}\right)$. Preparation of the medium followed the $\mathrm{P}$ loading, desorption, and growing procedure from the method previously described by Coltman and Silva [14] [15] with some modifications.

In the first SAS experiment, activated alumina (AA) was loaded with six $\mathrm{P}$ concentrations $\left(75,150,225,300,375\right.$, and $\left.450 \mathrm{mM} \mathrm{KH}_{2} \mathrm{PO}_{4}\right)$. Loaded AA was mixed with water-prewashed quartz sand (3\% w/w of AA) and transferred to SC10 cone-tainers (200 g per cone-tainer). Excess $\mathrm{P}$ was leached out with $60 \mathrm{ml}$ of deionized water daily for six days and then with $60 \mathrm{ml}$ of Clark's nutrient solution without $\mathrm{P}$ [12] for two days until the desired $\mathrm{P}$ media solution concentration was reached (S.1). EDDHA Fe chelate in form of Sprint 138 was added to the nutrient solution at the level of $75 \mathrm{mg} / \mathrm{L}$ that our preliminary testing showed optimal for sorghum under tested conditions. Based on the comparative plant performance at low and optimum Pi levels, potential candidates can be screened for P efficiency.

\section{Results and Discussion}

Exploration of sorghum MP root trait variability requires reproducible and accurate phenotyping approaches. Root phenotyping should be able to identify quantitative differences in RSA for deciphering underlying genetic mechanisms [16]. After exploring different root phenotyping platforms, our root trait phenotyping strategies can be summarized in Figure 2. We employed hydroponics and PGP for observation of the mutants' phenotypic variations in early stages (thick arrows). It allowed us to identify and systemize root traits in accordance to their potential contribution to abiotic stress resilience. Traits relevant to Pi efficiency 


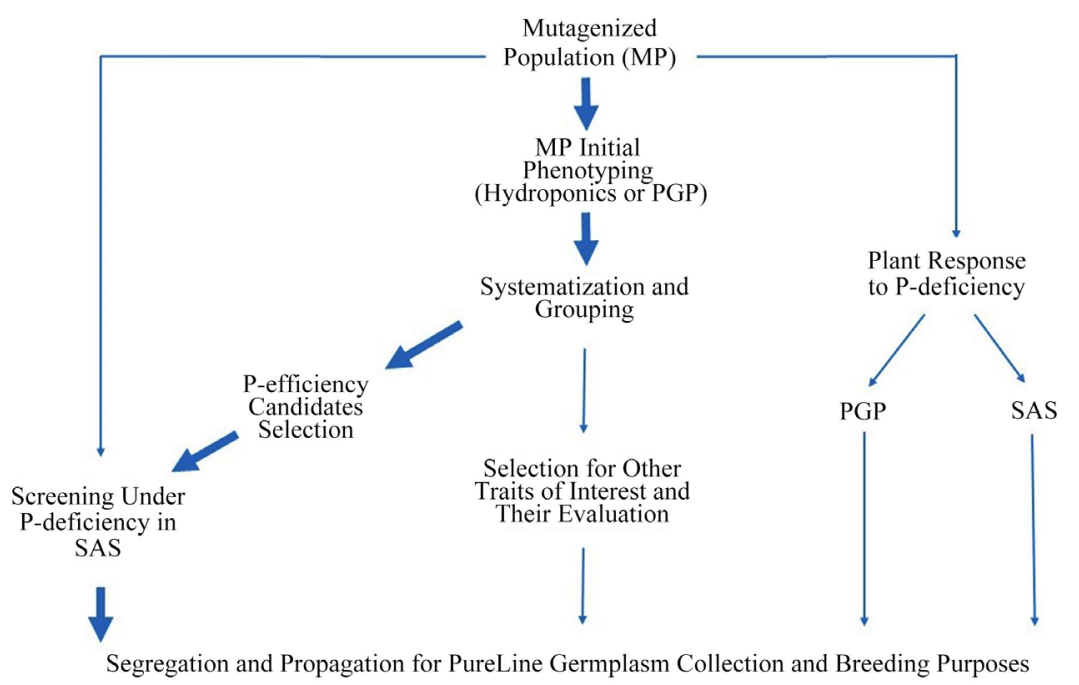

Figure 2. Phenotyping strategies for root traits.

are evaluated in the Pi availability modulated SAS comparing sorghum performance under deficient and optimal Pi levels. An alternative approach can be used in screening MP directly in SAS and then identifying the distinctive root traits contributing to superior plant performance. Despite the high labor and cost input, it may directly reveal lines with beneficial traits not identified in the preliminary PGP screening and should be considered for use in future large-scale Pi efficiency screening. Both techniques can also be used for studying root plasticity variability between genotypes in response to abiotic stresses.

These and other stress modulated environments can be used for selected traits evaluation in the degree of their contribution to stress adaptation. Subsequent segregation and propagation would allow for purification of specific mutations for breeding purposes.

\subsection{Mutagenized Population Phenotyping}

Hydroponics. MP grown hydroponically showed significant variability in root traits. Mutants were repeatedly examined for root traits distinctive from wild-type BTx623 and organized into groups based on percentage difference from the BTx623 (data not shown). We selected candidates with significant traits deviation from the original genotype that can be potentially relevant to P-efficiency such as LRL, root elongation in the upper $1 / 3$ of the primary root, intensive crown root development. Mutants were also inspected for unique morphological traits such as absence of dual primary root, significantly thicker primary root, absence of lateral roots, increased length of single lateral roots etc. (Figure 3). Selected mutants with traits of interest will be planted and grown for seed increase and used in further studies. Selection for specific trait variations and their segregation/purification in multiple generations can provide valuable genetic information and material for breeders. Even though the hydroponics method was used in our first screening efforts, PGP is proposed as alternative method with improved capabilities that is discussed in the next section. 


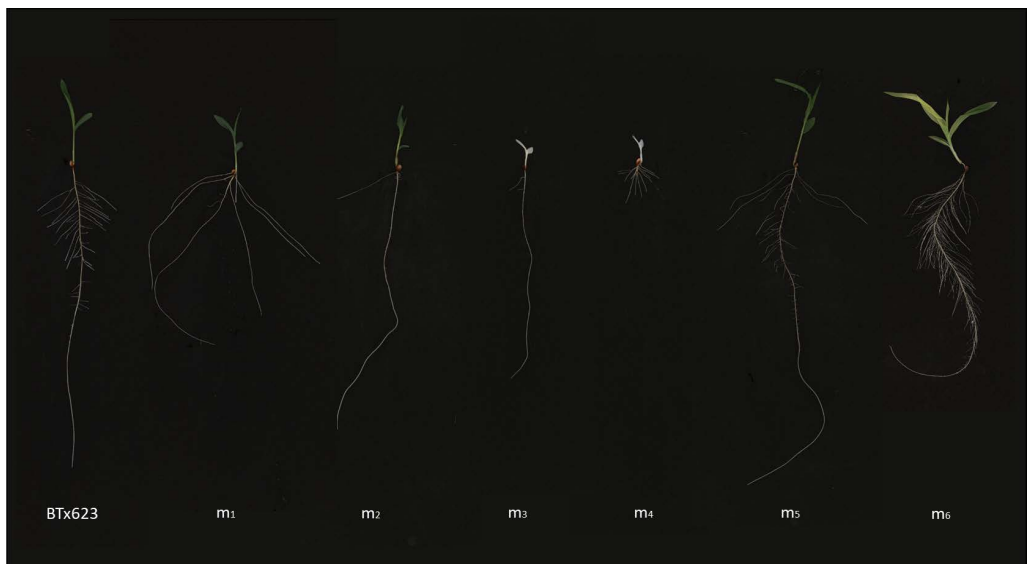

Figure 3. Distinct root morphology mutations observed during MP phenotyping. $\mathrm{m}_{1}$ : intensive crown and seminal root development, $\mathrm{m}_{2}$ : suppressed lateral root development, $\mathrm{m}_{3}$ : suppressed lateral root development and albinism, $\mathrm{m}_{4}$ : suppressed thick primary root development and albinism, $\mathrm{m}_{5}$ : intensive root growth in the upper $1 / 3$ of the primary root, $\mathrm{m}_{6}$ : intensive lateral root development, and BTx623-control.

Paper-based growth technique. Modified Hund paper-based growth pouch procedure provides inexpensive, space-saving, and high-throughput phenotyping of sorghum roots in the early stages of growth. It allows continuous root observation under multivariable nutrient availability conditions and acquisition of two-dimensional root images and their analysis [5] [17]. Compared to hydroponics, it provides improved roots aeration, lower seedling vulnerability to disease (especially molds), extended root growth, better imaging capabilities, and improved efficiency. Selected from hydroponics screening, MP candidate mutations have been grown on paper pouches to confirm distinct mutations in root architecture. It is proposed to be employed in the consequent root observation (instead of hydroponics) and Pi availability response studies in sorghum.

Selected candidates with traits assumed to be related to P efficiency will be evaluated in the subsequent plant performance analysis in the Pi controlled SAS (follows). This will provide evaluation of specific root trait contribution to $\mathrm{P}$ efficiency. In future studies, stratifying traits by their importance will allow us to focus on the most promising traits and develop/modify phenotyping method for their detailed observation and screening.

\subsection{Sorghum Root Response to Nutrients Availability.}

RSA development is shaped by plant's genetic composition and its interaction with environmental conditions [18]. While constitutive RSA traits are stable across various environments, plants can show significant degree of plasticity in response to stress conditions [19]. Root traits variability can increase in response to nutrient availability affecting root elongation, density, root hairs, and spatial location of roots etc. [20]. In this experiment, we tested sorghum plasticity in root growth response to nutrient availability in early stages of growth employing PGP technique. Sorghum was grown at a range of Pi availabilities at half and full strength nutrient solution to analyze qualitative and quantitative differences in 
root trait development.

In response to nutrient availability, plants developed distinct differences in multiple RSA traits even in first nine days of plant growth. Root responses to the combined nutrients (which encompasses complex aggregate response all nutrients) showed significant differences $(\mathrm{P}<0.05)$ (Table 1$)$ between treatments for TRL, PRL, LRL, and LRN (Figure 4). Some traits (such as TRL) showed strong correlation with nutrient solution concentration $\left(\mathrm{r}^{2}=0.96\right)$. Interestingly, at extreme nutrient deficiency (NS0), sorghum shifted allocation of limited resources to root growth (the ratio of RDW/SDW significantly increased). While TRL were the lowest in DI water due to reduced metabolic activity, primary roots and average lateral roots were longer (higher values of PRL and LRL/LRN) but thinner. Nutrient deficiency slowed new lateral root formation/emergence while favoring root elongation, allocating resources into media exploration.

Response to $\mathrm{Pi}$ availability appeared to be less pronounced compared to the response to combined nutrients which encompasses complex of aggregated responses to various nutrients (Figure 5). Among observed traits, TRL, LRL, and LRL/LRN significantly differed between treatments. Other differences appeared to be not significant on day 9; however, root trait variability may increase in later plant development. Some phenotypic variations attributed to plant's plasticity may be lower than constitutive root traits in early stages of growth [21]. Additional RSA responses to $\mathrm{P}$ deficiency may also develop in later stages with increasing need for $\mathrm{P}$ and plant timing triggering mechanisms for plasticity expression. PGP method is limited in growing length suggesting employing SAS or other techniques for this purpose.

Table 1. Means and standard deviations for root system architecture traits at early development.

\begin{tabular}{|c|c|c|c|c|c|c|c|c|c|c|c|c|c|c|c|c|c|c|c|c|c|}
\hline \multirow{2}{*}{\multicolumn{2}{|c|}{$\begin{array}{l}\text { Nutrien } \mathrm{P} \\
\text { Solution conc. } \\
\text { zonc. }(\% \text { (uM) }\end{array}$}} & \multicolumn{2}{|c|}{ TRLa } & \multicolumn{2}{|c|}{ PRL } & \multicolumn{2}{|c|}{ LRL } & \multicolumn{2}{|c|}{ PRL/LRL } & \multicolumn{2}{|c|}{ LRN } & \multicolumn{2}{|c|}{ LRL/LRN } & \multicolumn{2}{|c|}{$\mathrm{SDW}(\mathrm{mg})$} & \multicolumn{2}{|c|}{ RDW (mg) } & \multicolumn{2}{|c|}{ RDW/SDW } & \multicolumn{2}{|c|}{ 6LLR } \\
\hline & & Mean & s.d. & Mean & s.d. & Mean & s.d. & Mean & s.d. & Mean & s.d. & Mean & s.d. & Mean & s.d. & Mean & s.d. & Mean & s.d. & Mean & s.d. \\
\hline & 0 & 67.93 & 7.29 & 24.96 & 0.74 & 42.97 & 6.8 & 0.59 & 0.08 & 92 & 8.0 & 0.47 & 0.07 & 10.5 & 1.3 & 7.8 & 0.8 & 0.747 & 0.04 & 12.73 & 2.06 \\
\hline & 3 & 77.45 & 11.10 & 24.58 & 0.98 & 52.87 & 10.6 & 0.48 & 0.09 & 100 & 11.0 & 0.52 & 0.07 & 9.7 & 1.1 & 7.4 & 1.1 & 0.756 & 0.05 & 14.89 & 2.60 \\
\hline \multirow[t]{5}{*}{0.6} & 25 & 71.50 & 10.90 & 24.92 & 0.96 & 46.57 & 11.0 & 0.56 & 0.12 & 89 & 17.2 & 0.52 & 0.07 & 10.3 & 0.9 & 7.7 & 0.3 & 0.756 & 0.06 & 15.96 & 3.21 \\
\hline & 45 & 70.16 & 5.69 & 24.91 & 1.09 & 45.25 & 6.1 & 0.56 & 0.10 & 93 & 18.6 & 0.49 & 0.05 & 10.2 & 0.7 & 7.5 & 0.5 & 0.735 & 0.04 & 15.16 & 2.72 \\
\hline & 65 & 95.26 & 8.32 & 24.65 & 1.58 & 70.62 & 8.1 & 0.35 & 0.05 & 117 & 14.6 & 0.61 & 0.07 & 11.5 & 1.1 & 8.7 & 0.7 & 0.761 & 0.03 & 17.02 & 2.16 \\
\hline & 0 & 75.72 & 11.90 & 24.62 & 0.73 & 51.11 & 11.7 & 0.51 & 0.12 & 92 & 11.4 & 0.55 & 0.09 & 10.0 & 0.5 & 8.4 & 0.5 & 0.844 & 0.05 & 14.74 & 3.23 \\
\hline & 3 & 82.97 & 11.74 & 24.72 & 0.84 & 58.25 & 11.2 & 0.44 & 0.09 & 103 & 19.2 & 0.57 & 0.08 & 10.3 & 2.1 & 8.7 & 1.4 & 0.862 & 0.11 & 15.78 & 3.43 \\
\hline \multirow{3}{*}{1.0} & 45 & 75.89 & 10.13 & 24.95 & 0.71 & 50.95 & 10.7 & 0.51 & 0.12 & 103 & 10.1 & 0.50 & 0.10 & 10.2 & 0.7 & 8.0 & 0.5 & 0.787 & 0.04 & 12.86 & 2.07 \\
\hline & 65 & 84.50 & 13.50 & 25.05 & 0.68 & 61.83 & 13.2 & 0.44 & 0.09 & 114 & 21.1 & 0.53 & 0.09 & 10.3 & 0.7 & 8.7 & 0.6 & 0.844 & 0.04 & 15.76 & 2.04 \\
\hline & NS & \multicolumn{2}{|c|}{$0.048^{\star}$} & \multicolumn{2}{|c|}{$0.010^{*}$} & \multicolumn{2}{|c|}{$0.024^{*}$} & \multicolumn{2}{|c|}{$0.012^{*}$} & \multicolumn{2}{|c|}{$0.001^{\star}$} & \multicolumn{2}{|c|}{$0.049^{*}$} & \multicolumn{2}{|c|}{0.130} & \multicolumn{2}{|c|}{0.157} & \multicolumn{2}{|c|}{$0.001^{*}$} & \multicolumn{2}{|c|}{$0.038^{\star}$} \\
\hline \multirow[t]{2}{*}{$\mathrm{P}$ value } & $\mathrm{P}$ & \multicolumn{2}{|c|}{$0.004^{\star}$} & \multicolumn{2}{|c|}{0.9380} & \multicolumn{2}{|c|}{$0.002^{*}$} & \multicolumn{2}{|c|}{0.064} & \multicolumn{2}{|c|}{0.064} & 0.0 & $39 *$ & 0. & & 0.0 & & 0.9 & & & \\
\hline & $\mathrm{NS}^{*} \mathrm{P}$ & 0.3 & & 0.90 & 062 & 0.2 & & & & 0.2 & 93 & & & & & & & 0.2 & 43 & 0.2 & \\
\hline
\end{tabular}

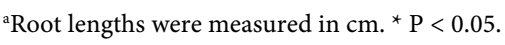




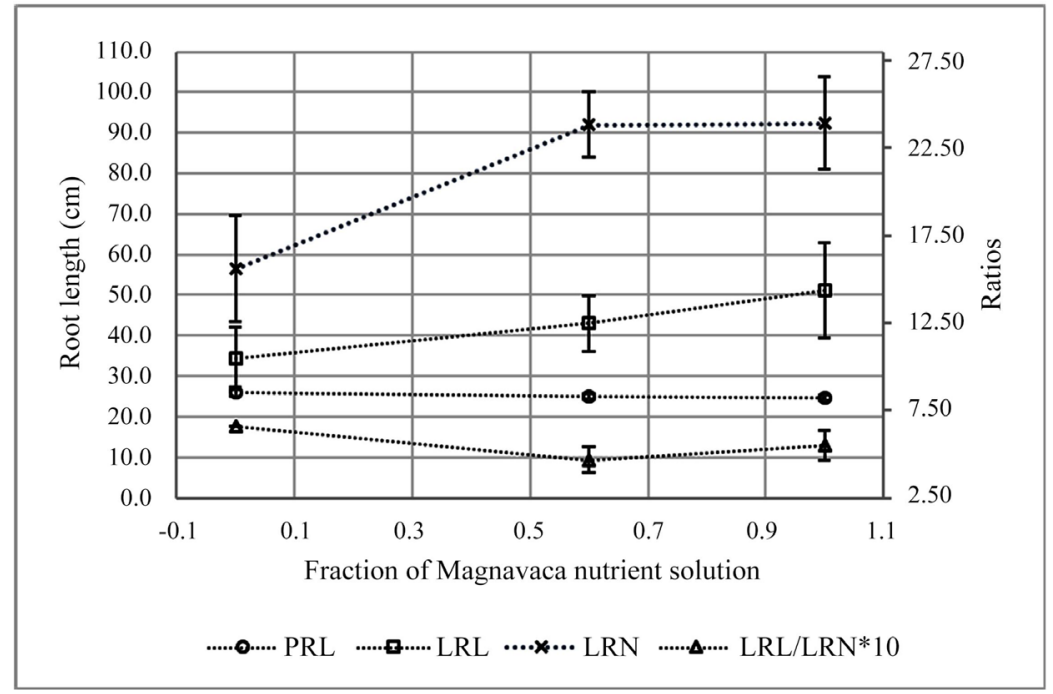

Figure 4. Primary and lateral root growth in response to nutrients availability. PRL: primary root length, LRL: lateral root length, LRN: lateral root number, LRL/LRN: average lateral root length.

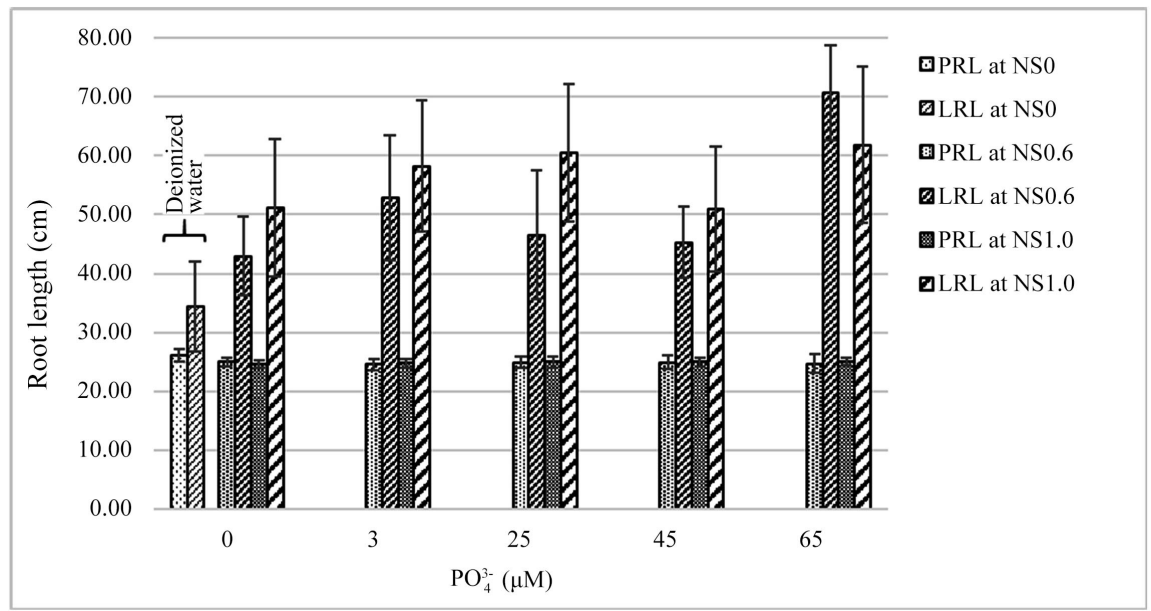

Figure 5. Primary and lateral root growth in response to Pi availability.

Similar to the nutrient solution, higher Pi availability increased the TRL and LRL while keeping PRL growth rate constant. Primary roots play a minor role in $\mathrm{P}$ acquisition that can justify reduced PR growth at low Pi availability; although monocot species and genotypes vary in their adaptive PR response to low $\mathrm{P}$ [22].

Lower Pi response flexibility could be related to prevailing constitutive adaptations in sorghum. Sorghum evolved predominantly in low-P soils that excluded advantages of developing RSA plasticity. In contrast, larger variation in other soil nutrients created premises for developing root plasticity to specific nutrition environments giving them competitive advantages.

Response evaluation of the original BTx623 sorghum inbred line lays groundwork for the subsequent phenotyping of the EMS-mutagenized population and the potential for expanding our understanding of genetic regulation of plant responses to nutrient availability. Paper pouches can be a valuable ap- 
proach used in further study of root plasticity variations of sorghum cultivars with potential adaptations to abiotic stresses.

\subsection{P-Loaded Sand-Alumina Culture in Sorghum P-Efficiency Improvement}

The above-described approaches can provide valuable information on root anatomy and physiology and reveal constituent or plastic genetic mechanisms regulating them. This gives basis for the selection of candidates with desired root traits influencing $\mathrm{P}$ efficiency. Subsequent validation for the performance of plants with specific root traits can be done on P-deficient soils in the field or greenhouse. The low reproducibility (due to the high level of environment-soil-root relationship complexity) and high cost make this approach very challenging. A promising technique is a solid-phase sand-alumina system (SAS). This system can simulate soil temporal and special $\mathrm{P}$ distribution, localized $\mathrm{P}$ depletion, and slow release and diffusion limited $\mathrm{P}$ behavior conditions in soils but other controlled-environment growing techniques (hydroponics, agar etc.) can't provide [15] [23] [24]. SAS can reproduce closely to natural P-deficient soil conditions while reducing the confounding effect of environmental factors and providing single-variable conditions [14]. It can maintain relatively stable Pi concentration (S-1) enabling comparison of plant performance at various Pi levels. At the same time, ability to manage Pi concentration in the media opens opportunities for modulation of the special phosphorus availability that simulates specific natural Pi allocation in soils (e.g. higher Pi content in upper soil horizons). Ease of separation of the sand from roots allows extended growth/observation period (more than nine days) using larger containers, thereby enhancing better development of the response traits. Even though the PGP technique can be successfully used to analyze plasticity in plant response to P-deficiency, it does not represent the natural soil environment which can limit development and identification of all adaptation mechanisms [25]. SAS, providing close to natural conditions and better control over environmental factors, allows us to capture more accurately plant response flexibility to Pi availability.

Based on the sorghum performance at six different Pi concentrations (Table 2 and Figure 6), deficient and optimal Pi levels (150 mM and $375 \mathrm{mM}$ P loading concentrations) were selected for sorghum growth comparison. In our subsequent study, candidates selected from an EMS-mutagenized population (or other cultivars of interest) with sufficient/deficient RSA traits will be tested for performance in SAS. The further study will evaluate the specific traits and determine their roles in $\mathrm{P}$ acquisition allowing us to narrow down our phenotyping and selection focus.

\section{Perspectives and Conclusions}

This EMS-mutagenized population is a valuable and useful genetic source for identifying genes responsible for abiotic adaptations [10]. Such efforts are restrained by limited root phenotyping data. The proposed phenotyping platform 
Table 2. Plant shoot dry weight at six p-loading treatments.

\begin{tabular}{|c|c|c|c|c|c|c|c|}
\hline \multirow{2}{*}{ Measured variables } & \multicolumn{7}{|c|}{ Loading $\mathrm{P}$ concentration (mM) } \\
\hline & 0 & 75 & 150 & 225 & 300 & 375 & 450 \\
\hline $\begin{array}{c}\mathrm{PO}_{4}^{3-} \text { concentration in } \\
\mathrm{SAS} \pm \text { s.d. }(\mu \mathrm{M})\end{array}$ & $0.0 \pm 0.15$ & $0.11 \pm 0.32$ & $3.84 \pm 0.53$ & $12.77 \pm 1.70$ & $21.27 \pm 0.69$ & $42.97 \pm 1.68$ & $58.82 \pm 4.62$ \\
\hline $\begin{array}{l}\text { Average shoot dry } \\
\text { weight } \pm \text { s.d. (g) }\end{array}$ & $0.0307 \pm 0.0099$ & $0.0463 \pm 0.0152$ & $0.0567 \pm 0.0225$ & $0.0640 \pm 0.0271$ & $0.0935 \pm 0.0091$ & $0.1055 \pm 0.0404$ & $0.1170 \pm 0.0455$ \\
\hline
\end{tabular}

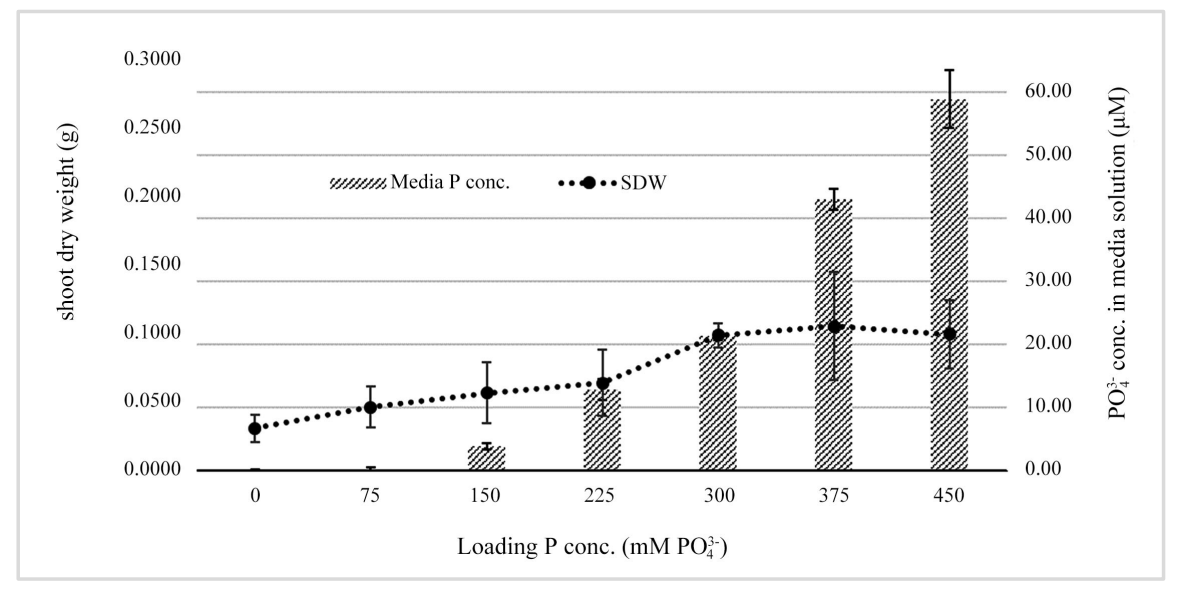

Figure 6. Plant shoot dry weight at six phosphorus loading concentrations.

employs a combination of phenotyping methods varying in the length, focus, capabilities to manipulate variables, cost efficiencies, and serving different yet complementary roles.

In the initial discovery phase involving high-throughput phenotyping of root variability, PGP is a method of choice that has been used in phenotyping EMS-mutagenized populations and is suggested to be used for other plant germplasm collections. Efficiency and flexibility of the PGP method allow us to utilize multiple forward and reverse genetics approaches. Ongoing phenotyping, stratifying and association analysis of genetic variations in root traits will allow us to identify QTLs associated with root traits. Growing information on germplasm sequencing and the identification of mutations extends opportunities to decipher gene function particularly that are unique to sorghum. Ability of the PGP approach to manipulate multiple factors, such as Pi availability, allows for exploration of adaptation mechanisms and genetics regulating plasticity of sorghum response to abiotic stress. Selection of candidates with constitutive and plastic root traits of interest with high heritability and subsequent segregation for desired root traits in further generations to produce homologous pure lines will further facilitate trait integration into commercial cultivars.

Contribution of root traits to adaptation efficiency is currently evaluated through exposing candidates with selected root traits to reproducible close to natural stress conditions and assessment of resulting plant performance in SAS. It has demonstrated ability to evaluate phenotypes for their role and degree of 
contribution to P-efficiency under low Pi availability. Plant assessment in later stages will allow us to correlate plant performance and yield with traits observed in early growing stages and help in developing markers for better selection. Prioritizing traits will help us to narrow down our focus in developing and applying specific, detailed phenotyping approaches thereby improving selected mutant screenings.

\section{Acknowledgements}

The authors would like to thank Ms. Sarah Moon for her excellent assistance in conducting hydroponics and PGP experiments.

\section{Conflicts of Interest}

The authors declare no conflicts of interest regarding the publication of this paper.

\section{References}

[1] Rakshit, S. and Wang, Y.-H. (2016) The Sorghum Genome. Springer, Berlin. https://doi.org/10.1007/978-3-319-47789-3

[2] Rooney, W. (2018) Achieving Sustainable Cultivation of Sorghum; Volume 1: Genetics, Breeding and Production Techniques. Burleigh Dodds Science Publishing Limited, Cambridge. https://doi.org/10.1201/9781351114462

[3] Trachsel, S., Kaeppler, S.M., Brown, K.M. and Lynch, J.P. (2011) Shovelomics: High Throughput Phenotyping of Maize (Zea mays L.) Root Architecture in the Field. Plant \& Soil, 341, 75. https://doi.org/10.1007/s11104-010-0623-8

[4] Klepper, B. and Kaspar, T. (1994) Rhizotrons: Their Development and Use in Agricultural Research. Agronomy Journal, 86, 745-753. https://doi.org/10.2134/agronj1994.00021962008600050002x

[5] Hund, A., Trachsel, S. and Stamp, P. (2009) Growth of Axile and Lateral Roots of Maize: I Development of a Phenotying Platform. Plant and Soil, 325, 335-349. https://doi.org/10.1007/s11104-009-9984-2

[6] Atkinson, J.A., Wingen, L.U., Griffiths, M., Pound, M.P., Gaju, O., Foulkes, M.J., Le Gouis, J., Griffiths, S., Bennett, M.J. and King, J. (2015) Phenotyping Pipeline Reveals Major Seedling Root Growth QTL in Hexaploid Wheat. Journal of Experimental Botany, 8, 2283-2292. https://doi.org/10.1093/jxb/erv006

[7] Amy, M.B., Jonathan, L. and Sieglinde, S. (1996) Effect of Phosphorus Deficiency on Growth Angle of Basal Roots in Phaseolus vulgaris. The New Phytologist, 132, 281. https://doi.org/10.1111/j.1469-8137.1996.tb01847.x

[8] Joshi, D.C., Singh, V., Hunt, C., Mace, E., van Oosterom, E., Sulman, R., Jordan, D. and Hammer, G. (2017) Development of a Phenotyping Platform for High Throughput Screening of Nodal Root Angle in Sorghum. Plant Methods, 13, 56. https://doi.org/10.1186/s13007-017-0206-2

[9] Xin, Z., Wang, M.L., Barkley, N.A., Burow, G., Franks, C., Pederson, G. and Burke, J. (2008) Applying Genotyping (TILLING) and Phenotyping Analyses to Elucidate Gene Function in a Chemically Induced Sorghum Mutant Population. BMC Plant Biology, 8, 103. https://doi.org/10.1186/1471-2229-8-103

[10] Jiao, Y., Burke, J., Chopra, R., Burow, G., Chen, J., Wang, B., Hayes, C., Emendack, 
Y., Ware, D. and Xin, Z. (2016) A Sorghum Mutant Resource as an Efficient Platform for Gene Discovery in Grasses. The Plant Cell, 28, 1551-1562. https://doi.org/10.1105/tpc.16.00373

[11] Magnavaca, R., Gardner, C.O. and Clark, R.B. (1987) Evaluation of Inbred Maize Lines for Aluminum Tolerance in Nutrient Solution. Genetic Aspects of Plant Mineral Nutrition: Proceedings of the Second International Symposium on Genetic Aspects of Plant Mineral Nutrition, Madison, 16-20 June 1987, 255-265.

https://doi.org/10.1007/978-94-009-3581-5 23

[12] Clark, R.B. (1982) Nutrient Solution Growth of Sorghum and Corn in Mineral Nutrition Studies. Journal of Plant Nutrition, 5, 1039-1057. https://doi.org/10.1080/01904168209363037

[13] Clark, R.T., Famoso, A.N., Zhao, K., Shaff, J.E., Craft, E.J., Bustamante, C.D., McCouch, S.R., Aneshansley, D.J. and Kochian, L.V. (2013) High-Throughput Two-Dimensional Root System Phenotyping Platform Facilitates Genetic Analysis of Root Growth and Development. Plant, Cell \& Environment, 36, 454-466. https://doi.org/10.1111/j.1365-3040.2012.02587.x

[14] Coltman, R.R., Gabelman, W.H. and Gerloff, G.C. (1982) A Sand Culture System for Simulating Plant Responses to Phosphorus in Soil. Journal of the American Society for Horticultural Science, 107, 938-942.

[15] Silva, Á.E. and Gabelman, W.H. (1992) Screening Maize Inbred Lines for Tolerance to Low-P Stress Condition. Plant and Soil, 146, 181-187. https://doi.org/10.1007/BF00012011

[16] Paez-Garcia, A., Motes, C., Scheible, W.-R., Chen, R., Blancaflor, E. and Monteros, M. (2015) Root Traits and Phenotyping Strategies for Plant Improvement. Plants, 4, 334. https://doi.org/10.3390/plants4020334

[17] Le Marié, C., Kirchgessner, N., Marschall, D., Walter, A. and Hund, A. (2014) Rhizoslides: Paper-Based Growth System for Non-Destructive, High Throughput Phenotyping of Root Development by Means of Image Analysis. Plant Methods, 10, 13. https://doi.org/10.1186/1746-4811-10-13

[18] Malamy, J.E. (2005) Intrinsic and Environmental Response Pathways That Regulate root System Architecture. Plant, Cell \& Environment, 28, 67-77. https://doi.org/10.1111/j.1365-3040.2005.01306.x

[19] Collins, N.C., Tardieu, F. and Tuberosa, R. (2008) Quantitative Trait Loci and Crop Performance under Abiotic Stress: Where Do We Stand? Plant Physiology, 147, 469-486. https://doi.org/10.1104/pp.108.118117

[20] Paul, A.I., Jinming, Z., Aabid, S., Ian, W.D., Philip, N.B. and Tedd, E. (2012) High-Throughput Imaging and Analysis of Root System Architecture in Brachypodium Distachyon under Differential Nutrient Availability. Philosophical Transactions. Biological Sciences, 367, 1559. https://doi.org/10.1098/rstb.2011.0241

[21] Rogers, E.D. and Benfey, P.N. (2015) Regulation of Plant Root System Architecture: Implications for Crop Advancement. Current Opinion in Biotechnology, 32, 93-98. https://doi.org/10.1016/j.copbio.2014.11.015

[22] Péret, B., Desnos, T., Jost, R., Kanno, S., Berkowitz, O. and Nussaume, L. (2014) Root Architecture Responses: In Search of Phosphate. Plant Physiology, 166, 1713-1723. https://doi.org/10.1104/pp.114.244541

[23] Lynch, J. (1998) The Role of Nutrient-Efficient Crops in Modern Agriculture. Journal of Crop Production, 1, 241-264. https://doi.org/10.1300/J144v01n02 10

[24] Rendig, V.V. and Taylor, H.M. (1989) Principles of Soil-Plant Interrelationships. McGraw-Hill, New York. 
[25] Leiser, W.L., Rattunde, H.F.W., Piepho, H.-P., Weltzien, E., Diallo, A., Toure, A. and Haussmann, B.I.G. (2015) Phosphorous Efficiency and Tolerance Traits for Selection of Sorghum for Performance in Phosphorous-Limited Environments. Crop Science, 55, 1152-1162. https://doi.org/10.2135/cropsci2014.05.0392 